CASE REPORT

\title{
Anesthesia Management in VP Shunt Surgery in Neonates with Hypoplastic Left Heart Syndrome (HLHS) and Dandy Walker Syndrome
}

\author{
Purwoko ${ }^{*}$, Fandi Ahmad Muttaqin ${ }^{\otimes^{*}}$
}

Article Info :

Submitted :

17-03-2021

Accepted :

24-04-2021

Published :

26-04-2021

https://doi.org/10.20961/soj

$\underline{\text { a.v1i1.49476 }}$

Authors' affiliations :

*Department of

Anesthesiology and

Intensive Therapy, Medical

Faculty Universitas Sebelas

Maret, Surakarta, Indonesia

${ }^{\circledR}$ Correspondence:

fandiahmadmuttaqin@gmai

1.com

\begin{abstract}
Hypoplastic left heart syndrome (HLHS) is defined as the incomplete development of the left heart structures, including the mitral valve, left ventricle, aortic valve, and aortic arch. Delays in diagnosis and delay in intervention will increase the morbidity of neonates with HLHS. To understand the mechanism of HLHS along with the principles of anesthesia in neuroanesthetics procedures in pediatrics in order to obtain a good outcome. A 9day old baby girl, weight 2522 grams with HLHS (aortic atresia) with patent ductus arteriosus (PDA), type II atrial septal defect (ASD) with a left to right shunt, and dandy walker syndrome. The patient's head was enlarged from birth, no heart sounds were found, regular I-II heart sounds. The anesthetic approach in HLHS is to maintain preoperative hemodynamic stability by maintaining heart rate, preload, and PGE1, balancing systemic vascular resistance and pulmonary vascular resistance, preventing too high $\mathrm{PaO} 2$, and administering inotropic agents to increase cardiac output and keep the patent ductus arteriosus open. The balance of systemic and pulmonary blood flow is a key principle in the management of HLHS anesthesia. The main goal of HLHS anesthesia is to minimize hemodynamic changes to prevent compromised hemodynamics in both circulations and maintaining stability is essential in preventing morbidity, complications, and increasing good outcomes in surgery.
\end{abstract}

Keywords: hypoplastic left heart syndrome, dandy walker syndrome, VP-shunt 


\section{INTRODUCTION}

Hypoplastic left heart syndrome (HLHS) is defined as the development of an incomplete left heart structure, including the mitral valve, left ventricle, aortic valve, and aortic arch. ${ }^{1}$ HLHS contributes to $1.4 \%$ to $4.1 \%$ of all congenital heart diseases, with prevalence rates 2 to 3 cases per 10,000 live births in the United States, where the majority of cases are predominantly male, with a male to female ratio of $3: 2 .^{2}$ Approximately $90 \%$ of infants with left ventricular hypoplasia will die without surgical intervention. These deaths are caused by spontaneous closure of the ductus arteriosus, usually within the first month of life. ${ }^{3}$ About $30 \%$ of fetuses with HLHS have a genetic syndrome and / or a structural abnormality outside the heart. Many reports have linked the incidence of HLHS to chromosomal abnormalities, eg Turner syndrome ( $\mathrm{X}$ monosomy), trisomy 18, and Jacobsen syndrome. ${ }^{4}$ In a family-based study by Hinton et al. It was found that the heritability of HLHS was very high $(>0.9 \%) .^{5}$ The risk of recurrence of HLHS in families with 1 affected child is about $0.5 \%$ to $2 \%$. The risk of other congenital malformations in firstdegree family members has been reported to range from $10 \%$ to $17 \%$. This suggests the importance of performing echocardiographic screening of all first-degree family members of HLHS patients. ${ }^{2,4}$
HLHS consists of a spectrum of anatomical variants characterized by an undeveloped left side of the heart, which includes the mitral valve, left ventricular cavity, left ventricular outflow, aortic valve, ascending aorta, and aortic arch. ${ }^{2,3,6}$ in HLHS there can be 3 forms, consisting of aortic valve atresia with mitral valve atresia, aortic valve atresia with mitral valve hypoplasia, and aortic valve stenosis with mitral valve hypoplasia.

HLHS with aortic valve atresia and mitral valve atresia is the most extreme form of HLHS where the left ventricular cavity is very small and the ascending aorta and the aortic arch are extremely hypoplastic. This will cause no output flow from the left ventricle, so that the main source of systemic blood supply, namely from the right ventricle, will pass through the ductus arteriosus. Whereas in HLHS with aortic valve atresia with mitral hypoplasia, there will be hypoplasia of the ascending aorta and aortic arch and decreased systemic return flow only depends on the patent ductus arteriosus. The patent mitral valve and blood enter the left ventricle, but there is no way out. Left ventricular hypertrophy results from contractions with the atresia aortic valve. The thick left ventricle has an ischemic endomyocardial which can cause fibrotic tissue known as endocardial fibroelastosis. The hypoplastic left ventricular cavity may have a greater mass than the first 
HLHS subtype, resulting in distortion of the right ventricular inflow and insufficiency of the tricuspid valve. The third HLHS subtype, aortic valve stenosis with mitral valve hypoplasia, can cause blood to be unable to exit the left ventricle, which results in less hypoplasia of the left ventricle, ascending aorta, and aortic arch than the two previous HLHS subtypes. ${ }^{2,6}$

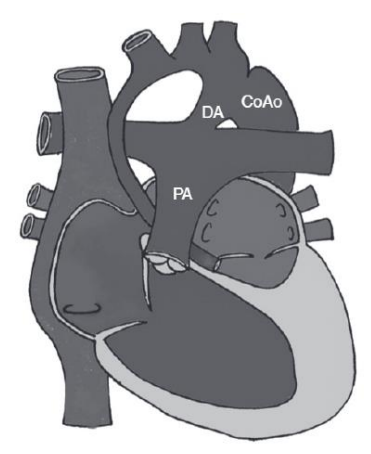

Figure 1. Anatomy of Hypoplastic Left Heart Syndrome (HLHS)

Yabrodi M, Mastropietro CW. Hypoplastic left heart syndrome: From comfort care to long-term survival. Pediatr Res. 2017;81(1-2):142-9.

At birth, children with HLHS rely on blood pumped by the right ventricle to the pulmonary artery (PA) to supply blood to the systemic circulation and end organs via a patent ductus arteriosus (PDA). In the majority of cases, HLHS is also accompanied by aortic coarctation (CoA) (Figure 1). ${ }^{1}$ In addition, HLHS is usually accompanied by a defect in the atrial septum, represented by the yellow arrow in Figure 2.

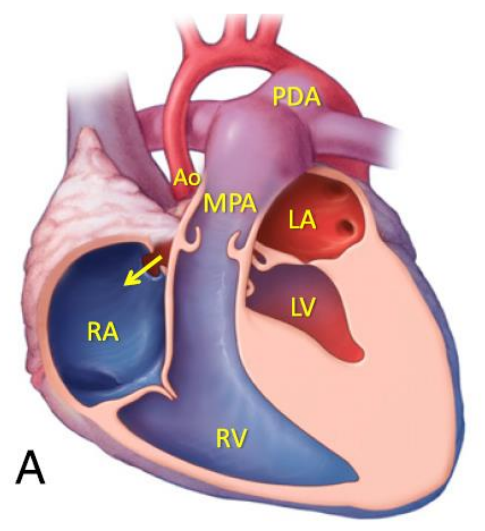

Figure 2. HLHS with the underdeveloped left ventricle.

Javed R, Cetta F, Said SM, Olson TM, O'Leary PW, Qureshi MY. Hypoplastic leftheart syndrome: An overview for primary care providers. Pediatr Rev. 2019;40(7):344-53.

HLHS can be diagnosed prenatally using obstetric ultrasound (USG) at weeks 18 to 24 . Ultrasound can identify congenital malformations associated with the 4 chambers of the heart, when the shape of the left side of the heart appears abnormal, echocardiography is indicated. During the fetal period, the left ventricle is filled by flow through the foramen ovale and restriction on atrial level shunting can cause damage to the left-sided heart structures during growth. As a result there is a change in the outflow or inlet of the left ventricle, which will lead to progression of left ventricular hypoplasia. ${ }^{7}$

\section{CASE ILLUSTRATION}

\section{Anamnesis}

A 9 days old baby girl weighing 2522 grams, has a large head complaint from birth. Complaints such as fever, cold cough, 
shortness of breath, chest pain, nausea, vomiting, seizures, blurred vision were denied. The patient had a history of birth with a caesarean section on the indication that the mother had a head-pelvic disproportion, with hydrocephalus non-comunicans, dandy walker syndrome, and asianotic congenital heart disease. There was no history of allergies, hypertension, diabetes mellitus, and asthma.

\section{Physical examination}

From the physical examination, we found that the patient looked moderately ill with GCS E4V5M6, pulse 130-135x / minute, respiratory rate $48 \mathrm{x} /$ minute, temperature $36.7{ }^{\circ} \mathrm{C}$, with $94-98 \% \quad \mathrm{SpO} 2$ with room air. Evaluation of free airway, open mouth, and mallampati is difficult to evaluate, free neck motion. On the head examination, a head circumference of $38 \mathrm{~cm}$ was found, a lump (-). Eye examination revealed anemic conjunctiva (- / -), icteric sclera (- / -), isocor pupil $(+/+)$, light reflex $(+/+)$, and corneal reflex $(+/+)$. On nasal examination, airway patency $(+/+)$, secretions (- / -), nostrils breath (-) were obtained, OGT was attached. On examination of the mouth, there was mucosal redness, no cyanosis was found. On lung examination, there was no abnormality. On cardiac examination, regular I-II heart sounds were obtained, the absence of noise. On examination of the abdomen, there was no abnormality. The patient had no DC, spontaneous urine products, warm in all four extremities, absence of edema in the superior and inferior limbs, and absence of cyanosis. On examination for VACTERL abnormalities, found a defect in the heart (C).

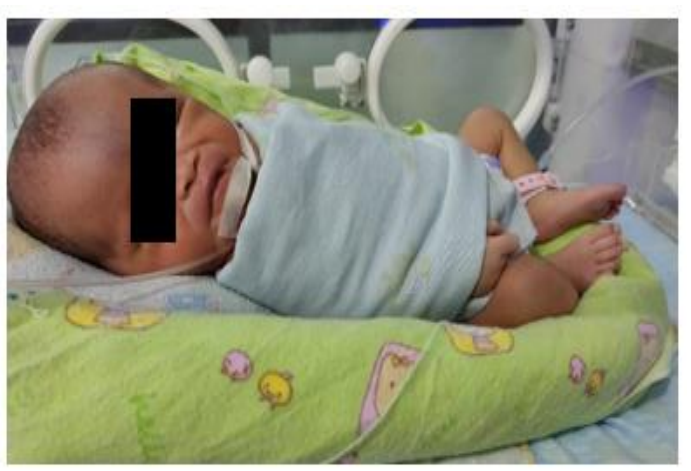

Figure 3. Clinical Photo Preoperative

\section{Supporting investigation}

Preoperative laboratory showed the value of hemoglobin $20.1 \mathrm{~g} \%$, hematocrit $55 \%$, leukocytes 19,900 / ul, PT 14.2 seconds, APTT 56.4 seconds, SGOT 24 U / L, SGPT $11 \mathrm{U} / \mathrm{L}$, total bilirubin $11.07 \mathrm{mg}$ / $\mathrm{dl}$, direct bilirubin $0.64 \mathrm{mg} / \mathrm{dl}$, and indirect bilirubin $10.43 \mathrm{mg} / \mathrm{dl}$, creatinine $2.0 \mathrm{mg} / \mathrm{dl}$, urea $67 \mathrm{mg} / \mathrm{dl}$, sodium $122 \mathrm{mmol} / \mathrm{L}$, and potassium $7.8 \mathrm{mg} / \mathrm{dl}$. Echocardiography shows ASD type II with left to right shunt, TR moderate PG $14.09 \mathrm{mmHG}$, atretic aorta without flow from the left ventricle to the aorta, wide PDA with flow from the 
pulmonary artery to the descending aorta, Large PDA with PA flow to Ao desc, Ao asec via PDA. Echocardiography results show Hypoplasia Left Heart Syndrome (HLHS). Thorax X-ray shows pneumonia and an enlarged heart with Left Ventricular Hypertrophy (LVH) configuration. MSCT Brain without contrast showed bilateral dilatation of the lateral ventricles and ventricles 3, with cystic lesions in the posterior fossa associated with the 4th ventricle, an enlarged posterior fossa with an inversion of lambdoidea, and cerebellar vermis hypoplasia with remnant vermi rotation, with features of Dandy-Walker malformation and non-communicating hydrocephalus.

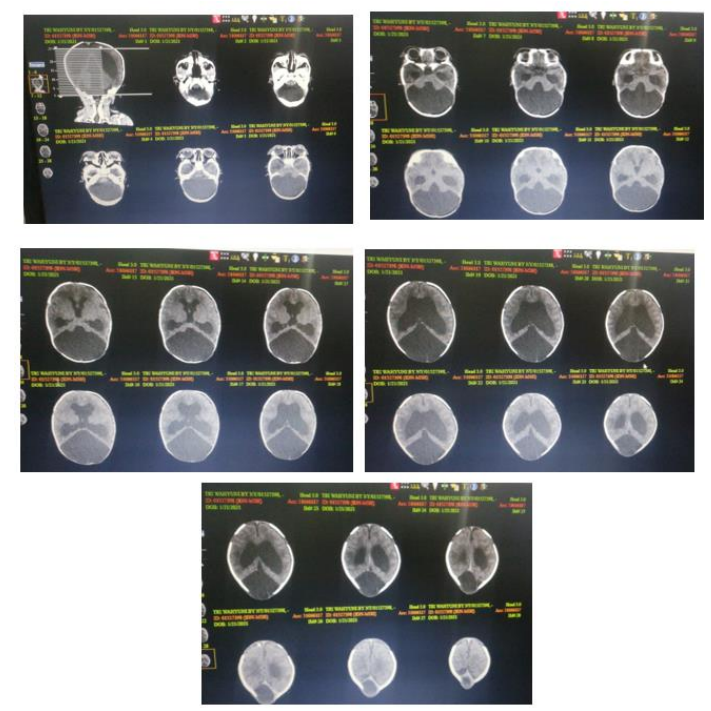

Figure 4. MSCT Brain Without Contrast

\section{Anesthesia Management}

After obtaining consent from the family and there is no contraindication for pediatric neuroanesthesia in the form of VP Shunt. Preoperative management in the form of fasting and intravenous fluid administration is sufficient with D5 1/4 NS 5cc / hour by infusion pump, this is aimed at keeping SVR and PVR balanced. In the operating room, a standard monitor was installed according to the ASA recommendations. The patient's hemodynamics during surgery was quite stable, with a blood pressure of $65 / 35 \mathrm{mmHg}$, a pulse rate of $145-150 \mathrm{x} / \mathrm{min}$, and $\mathrm{SpO} 2$ of 95-100\%. Patients induced anesthesia with intravenous ketamine $2.5 \mathrm{mg}(1 \mathrm{mg} / \mathrm{kg})$, Fentanyl IV $0.5 \mathrm{mcg}$, maintenance anesthesia was performed using Sevoflurane gas $2 \mathrm{v} \%$ in FiO2 $60-80 \%$ versus airbar. The patient was also given a muscle relaxant in the form of Atracurium $0.4 \mathrm{mg} / \mathrm{kg}$ intravenously (1mg) before the endotracheal tube no. 3.0 oral nonkinking.

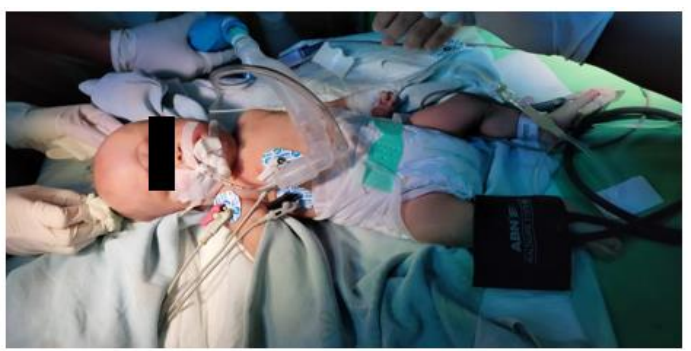

Figure 5. Clinical photo during surgery 
Anesthesia was maintained with controlled ventilation Sevoflurane 1-2.5 v\% in $50-60 \%$ oxygen versus airbar. The patient had transient arrhythmias and bradycardia at initial induction then stabilized back to the baseline vital sign preoperatively. During the operation the patient is stable and the operation lasts for 1 hour with a pulse rate ranging from 131-150 x / minute, systolic blood pressure in the range of $55-68 \mathrm{mmHg}$, SpO2 94-97\%. The total fluid intake during operation is $10 \mathrm{cc} /$ hour via an infusion pump. Operation bleeding was found to be $5 \mathrm{cc}$.

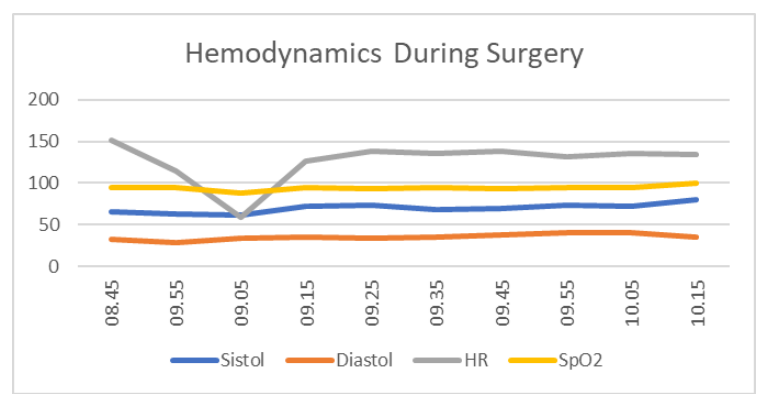

Figure 6. Hemodynamic Charts During Surgery

After confirming the muscle relaxant effect wears off, and the patient is hemodynamically stable, the patient is extubated in a conscious state and the patient is transferred to the NICU room.

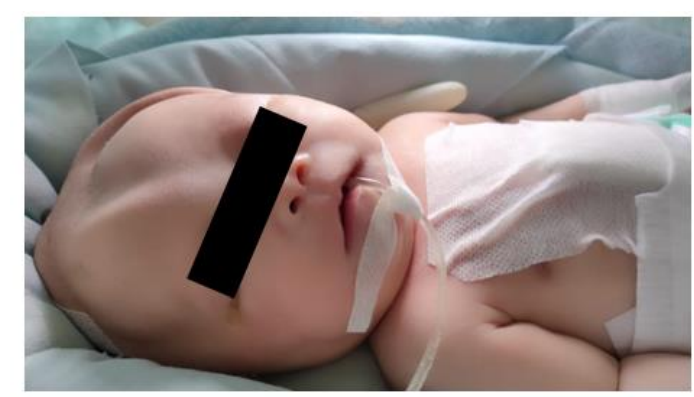

Figure 7. Clinical Photo Postoperative day-2

\section{DISCUSSION}

Comprehensive

preoperative assessment of these patients is necessary to determine the anatomic and physiological conditions and to identify signs of pulmonary and systemic flow dysfunction and signs of cardiac dysfunction. Preoperative management of patients with HLHS prioritizes systemic perfusion regulation so that blood flow to the lungs is the same as systemic blood flow (Qp: Qs). ${ }^{8}$ One of the common therapies to maintain ductal patency is the use of prostaglandin E1 which aims to maintain adequate systemic blood flow. After birth, pulmonary vascular resistance (PVR) will drop dramatically, so strategies are needed to achieve a balanced circulation, one of which is the use of intubation and mechanical ventilation. Ventilation techniques used include ventilation of room air or gas mixtures, or using $\mathrm{CO} 2$ aimed at increasing PVR. ${ }^{3,9}$ The most common manifestations of Qp: Qs imbalance are hypotension, lactic acidosis, and decreased urine production. ${ }^{9}$ Evaluation of Qp balance: Qs can be performed using near-infrared spectroscopy (NIRS) of the forehead and flank region non-invasively. ${ }^{3}$

The hemodynamic balance is determined by the PVR and SVR circulation and the goal of anesthesia is to minimize 
hemodynamic changes to prevent hemodynamic compromise in both circulations. Anesthesia for induction and maintenance can use volatile or intravenous agents. All anesthetic agents have the tendency to change hemodynamics and overuse can make the patient hemodynamically unstable. Anesthetic agents should be used carefully and titrated appropriately according to their hemodynamic effects. Positive pressure ventilation achieved by decreasing venous return can significantly reduce pulmonary blood flow in patients with passive pulmonary blood supply.

Anesthetic agents can decrease SVR resulting in increased systemic circulation and decreased pulmonary blood flow which can lead to hypoxemia. Conversely, a decrease in PVR will increase pulmonary blood flow which will decrease systemic perfusion and rapidly lead to a low cardiac output state. Oxygen supplementation will reduce PVR. ${ }^{1}$

The lowest possible oxygen concentration should be used during perioperative. If a high concentration of oxygen is required during induction and intubation, then high oxygen concentration should be reduced as soon as it is tolerated by the body. Hypocarbia and alkalosis will also reduce PVR and should be avoided. Increased afterload (SVR) should be avoided in consideration of the effects of pain, cold, distress, and vasopressor drugs. Hypotension after induction may occur and inotropes should be available if needed. ${ }^{1}$

Another principle is to keep pulmonary blood flow balance with the systemic flow (Qp: Qs) as close to 1: 1 as possible. This can be achieved by stabilizing cardiac output and using inotropic agents as needed. Peripheral oxygen saturation should be close to $80 \%$ followed by low inspired oxygen concentrations, this is to avoid hyperventilation with the inspiration of high oxygen concentrations which can cause hypocarbia and hyperoxia, both hypocarbia and hyperoxia can reduce PVR and increase the ratio of Qp: Qs>1. ${ }^{3}$

Use of inotropic agents aims for myocardial protection, with the use of dopamine 3-5 $\mu \mathrm{g} / \mathrm{kg} / \mathrm{min} .{ }^{9}$ The commonly used inotropic uses are milrinone, epinephrine, and dopamine. ${ }^{11}$ Milrinone has been shown to be safe to avoid low cardiac output in children after cardiac surgery. ${ }^{11,12}$ However, some investigators have found an impaired increase in oxygen consumption in neonates after the Norwood procedure in HLHS patients with dopamine use. ${ }^{13}$ Complications of anesthesia in patients with HLHS include arrhythmias, myocardial ischemia, shunt thrombosis, low cardiac 
output states, infection, prolonged mechanical ventilation, and cardiac arrest. ${ }^{1}$ Therefore, planning the selection of inotropic agents and dosages is essential for good clinical outcomes in patients after surgery.

\section{CONSLUSION}

Anesthetic management in patients with HLHS consists of preoperative, intraoperative and postoperative management. Preoperative management aims to ensure the pulmonary blood flow is equal to systemic. Hemodynamic balance is determined by circulating PVR and SVR. The goal of anesthesia is to minimize hemodynamic changes in order to prevent compromised hemodynamics in both circulations and to prevent morbidity, complications and increase good outcome in surgery.

\section{REFERENCE}

1. Yabrodi M, Mastropietro CW. Hypoplastic left heart syndrome: From comfort care to long-term survival. Pediatr Res. 2017;81(1-2):142-9.

2. Javed R, Cetta F, Said SM, Olson TM, O'Leary PW, Qureshi MY. Hypoplastic leftheart syndrome: An overview for primary care providers. Pediatr Rev. 2019;40(7):344-53.

3. Twite MD, Ing RJ. Anesthetic considerations in infants with hypoplastic left heart syndrome. Semin Cardiothorac Vasc Anesth. 2013;17(2):137-45.

4. Benson DW, Martin LJ, Lo CW. Genetics of hypoplastic left heart syndrome. J Pediatr. 2016;173:25-31.

5. Grossfeld PD. Hypoplastic Left Heart Syndrome. It Is All in the Genes**Editorials published in the Journal of the American College of Cardiology reflect the views of the authors and do not necessarily represent the views of JACC or the American College of Cardiology. J Am Coll Cardiol [Internet]. 2007;50(16):15967. Available from: http://www.ncbi.nlm.nih.gov/pubmed/179 36160

6. Tchervenkov CI, Jacobs JP, Weinberg PM, Aiello VD, Béland MJ, Colan SD, et al. The nomenclature, definition and classification of hypoplastic left heart syndrome. Cardiol Young. 2006;16(4):339-68.

7. Mohanty SR, Patel A, Kundan S, Radhakrishnan HB, Rao SG. Hypoplastic left heart syndrome: current modalities of treatment and outcomes. Indian $\mathrm{J}$ Thorac Cardiovasc Surg [Internet]. 2021 Jan 11;37(S1):26-35. Available from: http://link.springer.com/10.1007/s12055019-00919-7

8. Naguib AN, Winch P, Schwartz L, Isaacs J, Rodeman R, Cheatham JP, et al. Anesthetic management of the hybrid stage 1 procedure for hypoplastic left heart syndrome (HLHS). Pediatr Anesth 
[Internet]. $2010 \quad J a n ; 20(1): 38-46$.

Available from:

http://doi.wiley.com/10.1111/j.1460-

9592.2009.03205.x

9. Baker-Smith CM, Neish SR, Klitzner TS, Beekman RH, Kugler JD, Martin GR, et al. Variation in Postoperative Care following Stage I Palliation for Single-ventricle Patients: A Report from the Joint Council on Congenital Heart Disease National Quality Improvement Collaborative. Congenit Heart Dis. 2011;6(2):116-27.

10. Hoffman TM, Wemovsky G, Atz AM, Kulik TJ, Nelson DP, Chang AC, et al. Efficacy and safety of milrinone in preventing low cardiac output syndrome in infants and children after corrective surgery for congenital heart disease. Circulation. 2003;107(7):996-1002.

11. Li J, Zhang G, Holtby H, Humpl T, Caldarone CA, Van Arsdell GS, et al. Adverse Effects of Dopamine on Systemic Hemodynamic Status and Oxygen Transport in Neonates After the Norwood Procedure. J Am Coll Cardiol. 2006;48(9):1859-64. 